\title{
Nonequilibrium Quantum Condensation in an Incoherently Pumped Dissipative System
}

\author{
M. H. Szymańska, ${ }^{1}$ J. Keeling, ${ }^{2}$ and P. B. Littlewood ${ }^{3}$ \\ ${ }^{1}$ Clarendon Laboratory, Department of Physics, University of Oxford, Parks Road, Oxford, OX1 3PU, United Kingdom \\ ${ }^{2}$ Department of Physics, Massachusetts Institute of Technology, 77 Massachusetts Avenue, Cambridge, Massachusetts 02139, USA \\ ${ }^{3}$ Cavendish Laboratory, University of Cambridge, Madingley Road, Cambridge CB3 OHE, United Kingdom
}

(Received 5 March 2006; published 12 June 2006)

\begin{abstract}
We study spontaneous quantum coherence in an out of an equilibrium system, coupled to multiple baths describing pumping and decay. For a range of parameters describing coupling to, and occupation of the baths, a stable steady-state condensed solution exists. The presence of pumping and decay significantly modifies the spectra of phase fluctuations, leading to correlation functions that differ both from an isolated condensate and from a laser.
\end{abstract}

DOI: 10.1103/PhysRevLett.96.230602

The phenomenon of condensation, i.e., macroscopic occupation of a single quantum mode, has attracted much attention in recent years. It has been studied in systems such as atomic gases and superconductors [1], and ranges from the Bose-Einstein condensate (BEC) of structureless bosons, to the BCS type collective state of fermions, and intermediate states [2]. The next challenge is to control and study condensed states in solid state. Current candidates here include excitons in coupled quantum wells [3], microcavity polaritons [4-6], quantum Hall bilayers [7], and Josephson junction arrays in microwave cavities [8].

Unlike atomic gases, solid-state systems face dephasing and decay, as (with the special exception of superconductors) the condensate cannot usually be isolated from the environment: Phonons and impurities lead to dephasing, and due to imperfect trapping, particles escape, requiring external pumping to sustain a steady state. If such processes are faster than thermalization the system remains out of thermal equilibrium. Dissipation and decay not only present experimental obstacles, but also pose fundamental questions about the robustness of a condensate: Is a steadystate condensate possible with incoherent pumping and decay, if so, how does it differ from thermal equilibrium? Condensation in dissipative systems also provides a connection to the laser [9]. The relation between lasing and BEC is particularly relevant for polariton BEC, where the experimental distinction between the two is not simple [10].

Models that combine potentially strong nonequilibrium pumping with spontaneous symmetry breaking are not well studied, motivating study of simple models to extract the principal features. We study spontaneous condensation in a system coupled to independent baths, not in thermal or chemical equilibria with each other, providing incoherent pumping and decay. We focus on a Bose-Fermi system with disorder localized fermions; this is a model for exciton-polaritons [4-6] or Josephson junctions in microcavities [8]. However, many of our conclusions apply more generally to condensation with pumping and decay. We show that, despite changes to the excitation spectrum, and
PACS numbers: 05.70.Ln, 03.75.Gg, 03.75.Kk, 42.50.Fx

dephasing induced by pumping and decay, steady-state spontaneous condensation can occur in such systems, and can be distinct from lasing: The condensate can exist at low densities, far below inversion and lasing.

We study fluctuations about a steady-state condensate and find that the collective modes are qualitatively different from the modes found in thermal equilibrium [11]: Because of pumping and decay, the low energy phase mode (Goldstone, Bogoliubov mode) becomes diffusive at small momenta. By considering the effect of phase fluctuations, we find the decay of correlations, which at large times and distances differs both from that for a thermal equilibrium condensate and from a laser.

Our Hamiltonian is $\hat{H}=\hat{H}_{\text {sys }}+\hat{H}_{\text {sys,bath }}+\hat{H}_{\text {bath }}$, where,

$$
\begin{aligned}
\hat{H}_{\text {sys }}= & \sum_{\alpha} \epsilon_{\alpha}\left(b_{\alpha}^{\dagger} b_{\alpha}-a_{\alpha}^{\dagger} a_{\alpha}\right)+\sum_{\mathbf{p}} \omega_{\mathbf{p}} \psi_{\mathbf{p}}^{\dagger} \psi_{\mathbf{p}} \\
& +\frac{1}{\sqrt{L^{2}}} \sum_{\alpha} \sum_{\mathbf{p}}\left(g_{\alpha, \mathbf{p}} \psi_{\mathbf{p}} b_{\alpha}^{\dagger} a_{\alpha}+\text { H.c. }\right)
\end{aligned}
$$

describes two fermionic species $b_{\alpha}$ and $a_{\alpha}$, interacting with bosonic modes $\psi_{\mathbf{p}}$ normalized in a $2 \mathrm{D}$ box of area $L^{2}$, with $L \rightarrow \infty$, and coupled to three baths;

$$
\begin{aligned}
\hat{H}_{\text {sys,bath }}= & \sum_{\alpha, k} \Gamma_{\alpha, k}^{a}\left(a_{\alpha}^{\dagger} A_{k}+\text { H.c. }\right)+\Gamma_{\alpha, k}^{b}\left(b_{\alpha}^{\dagger} B_{k}+\text { H.c. }\right) \\
& +\sum_{\mathbf{p}, k} \zeta_{\mathbf{p}, k}\left(\psi_{\mathbf{p}}^{\dagger} \Psi_{k}+\text { H.c. }\right),
\end{aligned}
$$

given by $\quad \hat{H}_{\text {bath }}=\sum_{k} \omega_{k}^{\Gamma^{a}} A_{k}^{\dagger} A_{k}+\sum_{k} \omega_{k}^{\Gamma^{b}} B_{k}^{\dagger} B_{k}+$ $\sum_{k} \omega_{k}^{\zeta} \Psi_{k}^{\dagger} \Psi_{k}$. A single two-level system coupled to multiple baths has been recently studied in the context of the Kondo problem [12]. Condensed solutions of Eq. (1) have been studied in the context of atomic Fermi gases [13] and microcavity polaritons $[11,14]$. In this Letter we focus on microcavity polaritons, so $b_{\alpha}^{\dagger}, a_{\alpha}$ describe an electron and hole within a disorder-localized exciton state of energy $\epsilon_{\alpha}$. This can also be a fermionic representation of a hard-core boson or of a spin. These are dipole coupled to cavity photon modes $\psi_{\mathbf{p}}$, with low $\mathbf{p}$ dispersion, $\omega_{\mathbf{p}} \simeq \omega_{0}+\mathbf{p}^{2} / 2 m_{\mathrm{ph}}$, 
where $m_{\mathrm{ph}}=(\hbar / c)(2 \pi / w)$ is the photon mass in a $2 \mathrm{D}$ microcavity of width $w$. Because of the finite reflectivity of the cavity mirrors, photons escape, and so pumping (exciton injected) is required. Incoherent fermionic pumping and photon decay are described by (2), where $A_{k}, B_{k}$ are fermionic annihilation operators for the pump baths, while $\Psi_{k}$ are bosonic annihilation operators for photon modes outside the cavity.

With pumping and decay, the systems distribution must be obtained self consistently with the excitation spectrum. This prevents the use of imaginary time path integrals (e.g., [15]), as previously used to study the equilibrium properties of this model $[10,11,14]$. Instead, we use nonequilibrium Keldysh field theory, in a path-integral formulation [16]. Within this formalism, we integrate out the bath degrees of freedom and fermion fields to yield an effective description in terms of the photon field, parallel to that in $[10,11,14]$. The bath properties appear in this description via the functions $\kappa(\omega), \gamma(\omega), F_{A, B}(\omega)$, and $F_{\Psi}(\omega)$. The cavity decay rate $\kappa(\omega)=\pi \zeta^{2}(\omega) N^{\zeta}(\omega)$, where $\zeta$ is the coupling of the cavity photons to the bosonic modes in Eq. (2) and $N^{\zeta}$ is the density of states of these modes. Similarly $\gamma(\omega)=\pi \Gamma^{2}(\omega) N^{\Gamma}(\omega)$ where $\Gamma$ and $N^{\Gamma}$ are, respectively, coupling to, and the density of states of, the fermionic pumping baths. We assume a flat spectrum for the baths, so $\kappa$ and $\gamma$ are frequency independent. Frequency dependence is however present in the bath distribution functions; $F_{A, B}(\omega)=1-2 n^{A, B}(\omega)$, and $F_{\Psi}(\omega)=$ $1+2 n^{\Psi}(\omega)$, where $n^{A, B}, n^{\Psi}$ are occupations of the baths.

We proceed by looking for self-consistent solutions with a steady state, uniform, photon field of the form, $\psi(t)=$ $\psi_{0} e^{-i \mu_{s} t}$, giving a nonequilibrium generalization of the usual gap equation (e.g., [11,15]):

$$
\left(\omega_{0}-\mu_{S}-i \kappa\right) \psi_{0}=\sum_{\alpha} g_{\alpha} \int_{-\infty}^{\infty} d \omega i G_{\alpha, b a}^{K}(\omega) .
$$

Here, $i G_{\alpha, b a}^{K}\left(t, t^{\prime}\right)=\left\langle a_{\alpha, c l}^{\dagger}(t) b_{\alpha, c l}\left(t^{\prime}\right)\right\rangle$, is the anomalous Keldysh fermionic Green's function, where $a_{c l}=\left(a_{f}+\right.$ $\left.a_{b}\right) / \sqrt{2}$ and $f$ and $b$ are the forward and backward branches of the Keldysh time contour [16], and is given by:

$i G_{\alpha, b a}^{K}(\omega)=2 \gamma g_{\alpha} \psi_{0} \frac{\left(F_{A}+F_{B}\right) \omega+\left(F_{B}-F_{A}\right)\left(\tilde{\epsilon}_{\alpha}+i \gamma\right)}{\left[\left(\omega-E_{\alpha}\right)^{2}+\gamma^{2}\right]\left[\left(\omega+E_{\alpha}\right)^{2}+\gamma^{2}\right]}$,

where $E_{\alpha}=\sqrt{\tilde{\epsilon}_{\alpha}^{2}+g_{\alpha}^{2}\left|\psi_{0}\right|^{2}}, \tilde{\epsilon}_{\alpha}=\epsilon_{\alpha}-\mu_{S} / 2$, and the arguments of $F_{B, A}(\omega)$ are shifted by $\pm \mu_{S} / 2$ while $\omega$ is a real frequency. Note that we find that the bosonic bath's distribution $F_{\Psi}$ does not enter the mean-field gap equation, as the mean field does not consider the incoherent photons. In the limit $\gamma, \kappa \rightarrow 0$ with the bath distributions $F_{A, B}$ being thermal, Eq. (3) reduces to its equilibrium form $[11,14]$; for finite $\gamma$ and $\kappa$ it is significantly altered.

As in thermal equilibrium, the normal state $\psi_{0}=0$ is always a solution of Eq. (3), but for some range of parameters there is also a condensed $\psi_{0} \neq 0$ solution. When a solution $\psi_{0} \neq 0$ exists, the solution $\psi_{0}=0$ becomes un- stable. To understand this instability, we consider small fluctuations about the mean field, $\psi=\psi_{0}+\delta \psi$. The effective action for these fluctuations $\delta \psi$ has a part from the free photon action, and a part from interactions with fermions, $\frac{1}{2} \operatorname{Tr}\left(G \delta G^{-1} G \delta G^{-1}\right)$, where the fermionic Green's functions $G$ are $4 \times 4$ matrices in the Keldysh and particlehole $(a, b)$ spaces. Inverting the effective action for fluctuations gives the photon Green's functions, $\mathcal{D}^{K, R, A}$. The retarded and advanced Green's functions $\mathcal{D}^{R, A}$ give the excitation spectrum. The distribution function $F_{S}$, defined by $\mathcal{D}^{K}=\mathcal{D}^{R} F_{S}-F_{S} \mathcal{D}^{A}$, determines how the spectrum is occupied. As the system need not be in thermal or chemical equilibria with the baths, $F_{S}$ is in general not thermal, and differs from the bath distributions, $F_{A, B}$ and $F_{\Psi}$.

The instability of the normal state when a condensed solutions exists is analogous to thermal equilibrium. Even when the distribution $F_{S}$ is far from thermal, as the system approaches the phase transition, $F_{S}$ diverges at a frequency which we define as an effective chemical potential, where $\operatorname{Im}\left[\mathcal{D}^{R-1}\left(\mu_{\text {eff }}\right)\right]=0$. Taking the zeros of $\operatorname{Re}\left[\mathcal{D}^{R-1}\left(\omega^{*}, q\right)\right]$ as defining the normal modes of the system, condensation occurs when $\mu_{\text {eff }}$ reaches the bottom of the band of excitations, as in equilibrium. This condition, that a solution to $\mathcal{D}^{R-1}\left(\mu_{\mathrm{eff}}, q=0\right)=0$ exists, is equivalent to the gap equation, Eq. (3), at $\psi_{0}=0, \mu_{\text {eff }}=$ $\mu_{S}$, since $D^{R}$ describes a susceptibility which diverges at the transition. Beyond this point, the normal state is unstable, as $\mu_{\text {eff }}$ would lie in a bosonic band. This idea of instability can be directly connected to another: Beyond this point, the poles of the $\mathcal{D}^{R}$ have positive imaginary parts, fluctuations grow (rather than decay) in time. To see this, consider the imaginary parts of the poles of $\mathcal{D}^{R}$ as a function of momentum, $q$. At large $q$, these poles describe bare photons, and so are stable. By the previous definitions, above the transition, there is a $q$ at which the normal state Green's function has a real pole, $\mathcal{D}^{R-1}\left(\omega^{*}, q\right)=0$. The sign of the imaginary part of the pole changes at this point, so the low $q$ poles are unstable. Thus, a condensed solution to the gap equation implies instability of the $\psi_{0}=0$.

Unlike thermal equilibrium, there is however a range of parameters for which neither the normal state nor condensed steady-state solutions of the form $\psi(t)=$ $\psi_{0} e^{-i \mu_{s} t}$, are stable. This is not too surprising as systems similar to (1) are known to follow a complicated or even chaotic dynamics [17]. Although such anharmonic solutions would be of great interest, we focus here on steadystate condensed solutions of the usual form.

Understanding stability, we next solve the gap Eq. (3) to find the nonequilibrium phase diagram. Since Eq. (3) is complex it gives two equations for two unknowns: the order parameter $\psi_{0}$ and the frequency $\mu_{S}$. The common oscillation frequency $\mu_{S}$ would in thermal equilibrium be the system's chemical potential, considered as a control parameter, adjusted to match the required density, and the (real) gap equation determines only $\psi_{0}$. Here, as different baths have different chemical potentials, the system is not 
in chemical equilibrium with either bath, so both $\mu_{S}$ and $\psi_{0}$ must be found from the gap equation. The density, which can be found given $\psi_{0}$ and $\mu_{S}$, is set by the relative pump and decay strength.

Although the formalism would allow any distribution, from here, the baths are taken to be thermal, but not in equilibrium with each other, so the system may be far from thermal equilibrium. For simplicity, the figures are for the baths at zero temperature, so the bath distributions are defined by their chemical potentials, and the limit of a narrow bandwidth [14], $\epsilon_{\alpha}=\epsilon, g_{\alpha}=g$ is taken. To ensure that on average only one of the two fermionic levels $a, b$ is occupied, the chemical potentials of baths $A, B$ are related by $\mu_{A}=-\mu_{B}$. Since the bottom of the bulk photon band is far below the energy of the cavity photon modes, we take the chemical potential of the decay bath to be large and negative, measured from the bottom of the cavity photon band. Thus, the baths are described by three parameters, the couplings $\gamma, \kappa$ and $\mu_{B}$ parameterizing the occupation of the pumping baths.

Figure 1 shows a phase diagram in terms of $\gamma, \kappa, \mu_{B}$. It shows both where a condensed solution to the gap equation exists, and where the solution is stable. At a given $\kappa$ there is a minimum $\gamma$ (as pumping is proportional to $\gamma$ ) and a maximum $\gamma$ (as dephasing is proportional to $\gamma$ ) required for condensation. Stable condensed solutions exist only for $\kappa$ smaller than about $0.2 g$. In the region in Fig. 1 the condensed solutions are all below population inversion. However for $\gamma>g$, when in a weak-coupling regime, only laserlike solutions with population inversion are possible. For large $\gamma$ and $\mu_{B}$ (large pumping), our theory recovers the regular laser limit.

We now discuss the collective modes, considering fluctuations about the steady state. Motivated by microcavity polaritons, we study these collective modes by calculating the photoluminescence (PL) spectra: $i \mathcal{D}_{\psi^{\dagger} \psi}^{<}\left(t, t^{\prime}\right)=$

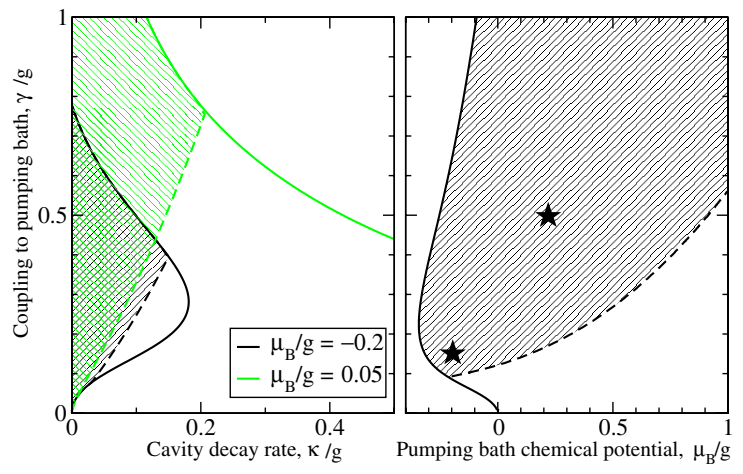

FIG. 1 (color online). Mean-field phase diagrams. Left: Critical $\gamma$ vs decay rate $\kappa$, for two different pumping bath chemical potentials, $\mu_{B}$. Right: Critical $\gamma$ vs $\mu_{B}$ (measured with respect to $\epsilon$ ) for $\kappa=0.038 \mathrm{~g}$ as in experiment [6]. Solid lines show where a solution of the gap equation exists, dashed lines show where that solution is stable. The region where a stable condensed solution exists is shaded. Stars mark the choice of parameters shown in Fig. 2. $\left\langle\psi_{f}^{\dagger}(t) \psi_{b}\left(t^{\prime}\right)\right\rangle$. In the normal state, as expected, one finds homogeneously broadened upper and lower polariton modes. This homogeneous broadening depends on all of $\kappa, \gamma, \mu_{B}$; it arises from the dephasing due to pumping and decay, and so appears naturally only in a nonequilibrium treatment. Approaching the phase boundary from the normal side, the lower polariton linewidth reduces to zero. This can be understood by identifying the zeros of the real part of the inverse Green's function as the polariton energies, $\operatorname{Re}\left[\mathcal{D}^{R-1}\left(\omega^{*}, q\right)\right]=0$, and the imaginary part as giving the linewidth, $1 / \tau_{p} \approx \operatorname{Im}\left[\mathcal{D}^{R-1}\left(\omega^{*}, q\right)\right]$. The earlier discussion relating the gap equation to $\mathcal{D}^{R-1}\left(\mu_{S}, q=\right.$ 0 ) implies that $1 / \tau_{p}$ vanishes at the transition.

When condensed, as in equilibrium [11], the spectrum changes. This leads to a soft mode, describing phase fluctuations, as global phase rotation symmetry is broken. As phase fluctuations may be large, one must include them to all orders [15] to find the field-field correlations; amplitude fluctuations remain small, as they have a restoring force. Writing, $\psi(t)=\sqrt{\rho_{0}+\pi(t)} e^{i \phi(t)}$, where $\rho_{0}$ is the meanfield condensate density, the PL spectrum is thus

$$
\begin{array}{r}
i \mathcal{D}_{\psi^{\dagger} \psi}^{<}(t, r)=\rho_{0}\left[1+O\left(1 / \rho_{0}\right)\right] \exp [-f(t, r)], \\
f(t, r)=\int d \nu \int(d q)^{2}\left[1-e^{i(\nu t+\mathbf{q} \cdot \mathbf{r})}\right] i \mathcal{D}_{\phi \phi}^{<}(\nu, q) .
\end{array}
$$

The phase-phase Green's function $\mathcal{D}_{\phi \phi}^{<}$is found by inverting the action expanded to quadratic order.

The $\mathcal{O}\left(1 / \rho_{0}\right)$ terms in Eq. (4), due to amplitudeamplitude and phase-amplitude Green's functions, are in-

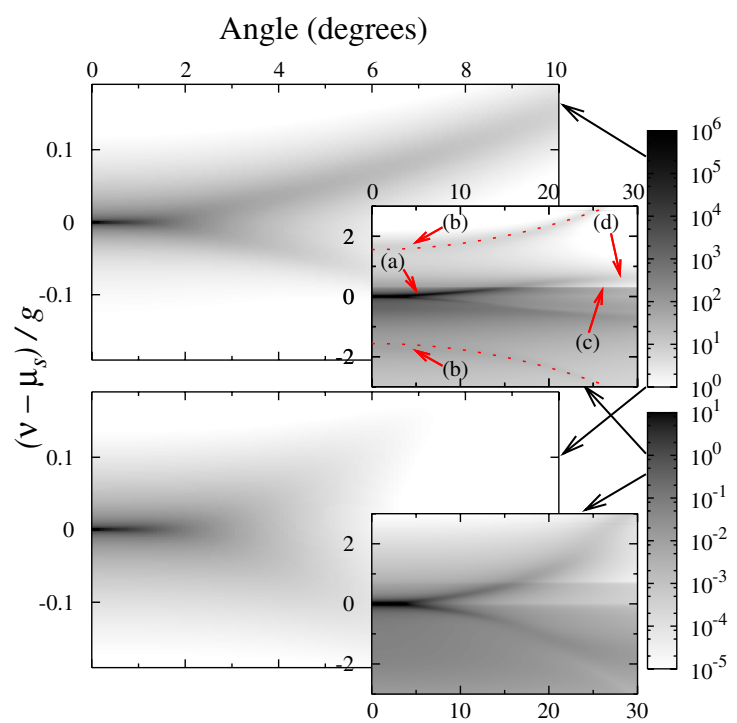

FIG. 2 (color online). Photoluminescence $i \mathcal{D}_{\psi^{\dagger} \psi}^{<}(\nu, q)$ of a condensed system, where $q$ is shown by angle of emission $\tan ^{-1}\left(\mathrm{cq} / \omega_{0}\right)$. Top: Strong coupling, Bottom: weak coupling, exact parameters marked by stars on Fig. 1. Main figures show PL from small $\nu, q$ region, to all orders in phase fluctuations. Insets show a larger range of $\nu, q$ for the same parameters. (Dotted lines have been added to show the faint amplitude mode). 
cluded when plotting Fig. 2, but their forms are complex, so not reproduced here. In the limit of small $v$ and $q, \mathcal{D}_{\phi \phi}^{<}$ takes a simple form:

$$
i \mathcal{D}_{\phi \phi}^{<}(\nu, q) \approx C\left[\left(c^{2} q^{2}-\nu^{2}\right)^{2}+4 \nu^{2} x^{2}\right]^{-1},
$$

where $C, c$, and $x$ can be found from the full expressions. Without pumping and decay, $x=0$. Nonzero $x$ means that rather than a linear dispersion at low $q$, as in a closed system in thermal equilibrium [11,15], the poles of the Green's function have the form $\nu=-i x \pm i \sqrt{x^{2}-c^{2} q^{2}}$. For $|q|<x / c$, these modes are diffusive; the poles are imaginary, only for $|q|>x / c$, do they acquire a real part. This behavior is apparent in the PL spectrum in Fig. 2. The diffusive behavior at small $q$ is not limited to Bose-Fermi systems; it should also be present in other condensed systems with pumping and decay. Note that at $q=0$ and $\nu=0, \mathcal{D}_{\phi \phi}^{<}$has a real pole; a manifestation of broken symmetry in the infinite system.

As given by Eq. (4), the PL spectrum does not depend linearly on $\mathcal{D}_{\phi \phi}^{<}$. If $\mathcal{D}_{\phi \phi}^{<}$were small, the exponential in Eq. (4) could be expanded, giving PL divided between a condensate term $\rho_{0} \delta(\nu) \delta(q)$, and a part from fluctuations. However, at $\nu, q \rightarrow 0, \mathcal{D}_{\phi \phi}$ is not small, and so phase fluctuations give a line shape to the condensate and determine long-time field-field correlations. In two dimensions, inserting Eq. (6) in Eq. (5), the main dependence of $f(t, r)$ on $t, r$ comes from a logarithmic divergence, $\int d q / q$, cut at large momenta by terms beyond those in Eq. (6), and at small momenta by one of $1 / r, 1 / c t, 1 / c \sqrt{t \tau_{g}}$ or $1 / c \tau_{g}$, where $\tau_{g}=1 / x$ is the lifetime of the phase mode. This logarithmic form leads to power law field-field correlations. [As in thermal equilibrium [15], where, $f(t, r)=$ $\eta \ln \left(\sqrt{c^{2} t^{2}+r^{2}} / \beta c\right)$, with $\eta \propto k_{B} T / \rho_{0}$.] According to the relative values of $r, t, \tau_{g}$, different lower cutoffs apply, and so the power law differs at different places in the $r, t$ plane. For small $r, t$ we recover the equilibrium power laws; but when $t \gg \tau_{g}$, and $c \sqrt{t \tau_{g}^{-}} \gg r$, then $\tau_{g}$ becomes important, and $f(t) \propto \ln \left(c \sqrt{t} \bar{\tau}_{g}\right)$, giving a condensate line shape that differs from a nondissipative, thermal equilibrium 2D system. Power law field correlations at long times lead to a power law divergence of their Fourier transform as $\nu, q \rightarrow 0$, so the linewidth is not well defined. This differs from phase diffusion for a single mode in laser theory [9], where phase correlations grow linearly in time, giving exponential decay of field correlations.

At higher $q$ and $\nu$ (Fig. 2 Insets) the difference between linearized in Green's functions and keeping all orders of phase fluctuations is not visible. The strong coupling spectrum has the usual phase and amplitude modes (marked $a, b)$ emerging from the lower and upper polariton branches. One can also see an occupation edge (c), and above that the lower polariton following the exciton dispersion (d). These higher $q$ features are analogous to thermal equilibrium nondissipative polariton condensation in strong coupling regime [11] but with nonthermal occu- pations. Increasing pumping, dephasing increases, and the system crosses to weak coupling (lower panels of Fig. 2), the polariton splitting is suppressed, and the spectrum follows the photon dispersion. The diffusive region at small momentum occurs both in strong and weak coupling and is a sign of condensation in the dissipative system.

To conclude, we have studied how steady-state spontaneous condensation emerges in nonequilibrium systems with pumping and decay. This condensation is distinct from lasing: It can occur at densities much lower than the population inversion and the decay of correlations, and thus condensate line shape, differ from that for phase diffusion of a single laser mode. However, this condensation also differs qualitatively from thermal equilibrium: Dissipation changes the spectrum, at low momenta, the phase mode is diffusive, changing the power-laws controlling long time decay of field-field correlations, and is expected to modify superfluidity. These conclusions, although studied here for microcavity polaritons, apply also to other Bose and Bose-Fermi condensates subject to pumping and decay.

We are grateful to B. D. Simons and R. Zimmermann for stimulating discussions. We acknowledge financial support from EPSRC (M. H. S.) and the Lindemann Trust (J. K.).

[1] Bose-Einstein Condensation, edited by A. Griffin, D. W. Snoke, and S. Stringari (Cambridge University Press, Cambridge, England, 1995).

[2] C. A. Regal, M. Greiner, and D. S. Jin, Phys. Rev. Lett. 92, 040403 (2004); M. W. Zwierlein et al., Phys. Rev. Lett. 92, 120403 (2004).

[3] L. V. Butov et al., Nature (London) 417, 47 (2002); 418, 751 (2002); D. Snoke et al., Nature (London) 418, 754 (2002).

[4] Le Si Dang et al., Phys. Rev. Lett. 81, 3920 (1998).

[5] H. Deng et al., Science 298, 199 (2002).

[6] M. Richard et al., Phys. Rev. Lett. 94, 187401 (2005).

[7] J.P. Eisenstein and A.H. MacDonald, Nature (London) 432, 691 (2004).

[8] P. Barbara et al., Phys. Rev. Lett. 82, 1963 (1999).

[9] H. Haken, in Quantum Optics, edited by Kay and Maitland (Academic, London, 1970), p. 201.

[10] M.H. Szymanska and P. B. Littlewood, Solid State Commun. 124, 103 (2002); M. H. Szymanska, P. B. Littlewood, and B. D. Simons, Phys. Rev. A 68, 013818 (2003).

[11] J. Keeling et al., Phys. Rev. Lett. 93, 226403 (2004); Phys. Rev. B 72, 115320 (2005).

[12] J. Paaske et al., Phys. Rev. B 70, 155301 (2004).

[13] M. Holland et al., Phys. Rev. Lett. 87, 120406 (2001); E. Timmermans et al., Phys. Lett. A 285, 228 (2001); Y. Ohashi and A. Griffin, Phys. Rev. Lett. 89, 130402 (2002).

[14] F. M. Marchetti et al., Phys. Rev. Lett. 96, 066405 (2006).

[15] N. Nagaosa, Quantum Field Theory in Strongly Correlated Electronic Systems (Springer-Verlag, Berlin, 1999).

[16] A. Kamenev, in Nanophysics: Coherence and Transport (Elsevier, New York, 2005), p 177.

[17] C. Emary and T. Brandes, Phys. Rev. Lett. 90, 044101 (2003). 